\title{
Urteile übers Tanzen aus der Reformationszeit.
}

\author{
Von OTTO CLEMEN.
}

"Tänze sind eingerichtet und zugestanden worden, damit artiges Benehmen gelernt werde im Verkehr, und Freundschaft und Bekanntschaft geschlossen werde unter Jünglingen und Mädchen. Denn so können die Charaktere erkannt werden, desgleichen bietet sich so Gelegenheit, in Ehren zusammenzukommen, so daß wir nach Prüfung des Sinnes des Mädchens ehrenhafter und sicherer freien können. Der Papst hat die Tänze öffentlich verdammt, weil er ein Gegner des Heiratens gewesen ist. Aber mit Maßen soll alles geschehen! Deshalb werden ehrliche Männer und Frauen eingeladen, die den Tänzen beiwohnen sollen, damit alles desto mehr in Ehren verläuft. Und ich werde bisweilen auch teilnehmen, damit, durch meine Gegenwart bewogen, die Jünglinge sich $\mathrm{zu}$ drehen aufhören." So äußerte sich Luther an seinem Tische in den Septembertagen des Jahres 1540, und Johann Mathesius, der damals wieder in Wittenberg weilte, um sich für das ihm angetragene Predigtamt in Joachimsthal vorzubereiten, hat die Rede aufgezeichnet. ${ }^{1}$ ) Wir sehen, wie unbefangen, weltoffen und menschlich und wie ernst und vernünftig anderseits Luther über das Tanzen und den Verkehr der beiden Geschlechter untereinander urteilt. Nur das Kreisen beim Tanzen gefiel ihm nicht. Auch anderwärts wurde es verurteilt: so erfahren wir aus dem ausführlichen und kulturgeschichtlich höchst interessanten Briefe, in dem Andreas Osiander aus Königs-

1) Luthers Tischreden in der Mathesischen Sammlung. Nach einer Handschrift der Leipziger Stadtbibliothek herausgegeben von Ernst Kroker, Leipzig 1903, S. 219, Nr. 411. Vgl. Corpus reformatorum XX, 578f. und de Wette-Seidemann, Luthers Briefe VI, 435. 
berg seinem Schwiegersohn Hieronymus Besold in Nürnberg von der Hochzeit seiner Tochter mit dem herzoglichen Leibarzt Andreas Aurifaber am 19. Januar 1550 berichtet, daß damals jeder, der sich im Kreise drehte, Strafe zahlen mußte. ${ }^{1}$ )

Noch weniger hatte Melanchthon, den wir uns doch sonst so leicht als überbedenklich und ängstlich vorstellen, am Tanzen etwas auszusetzen. Er mischte sich gelegentlich sogar selbst in den Reigen. Im Diarium des Johannes Rütiner von St. Gallen steht ein kurzer, freilich ziemlich verworrener Bericht über die Hochzeit des Franz Lambert von Avignon, die dieser in Wittenberg und zwar, wie wir aus anderen Quellen wissen, am 13. Juli 1523 feierte. Der Bericht gründet sich auf eine Erzählung des Johannes Keßler, der damals zu den Hochzeitsgästen gehörte. Hier heißt es: 3 choreas egerunt, Philippo saltante.?) Ferner schreibt Urban Balduyn, der bald darauf Stadtschreiber in Wittenberg wurde, unterm 1. August 1529 an den Zwickauer Stadtschreiber Stephan Roth: "Ey noch mehr, ich hab Melanchthonen mit der prebstin [d. i. Justus Jonas' Frau] sehen tantzen, es ist mir wunderlich gewesen." ${ }^{8}$ ) Und endlich meldet ein Brief vom Ende 1541 geradezu: solere ipsum interdum saltare.4)

Doch fehlte es nicht an gegnerischen Stimmen. So erschien

1) W. Mōller, Andreas Osiander, Elberfeld 1870, S. 343 f. Vgl. auch schon die bei R. Hofmann, Reformationsgeschichte der Stadt Pirna, Separatabdruck aus den Beiträgen zur sächsischen Kirchengeschichte VIII (1893), S. 29 aus dem Anfang des 16. Jahrhunderts angeführten Strafverfügungen gegen solche, die sich beim Tanze "verdreht" hatten, sowie die von $\mathrm{Fabian}$ in den Mitteilungen des Altertumsvereins für Zwickau und $\mathrm{Um}$ gegend II (4888), S. IX einer Zwickauer Polizeiverordnung von 1548 entnommenen Urteile gegen Ausschreitungen beim Rundtanze, endlich auch den hübschen Aufsatz von W us tmann, der Tanz in Leipzig in 15. und 16. Jahrhundert, Leipziger Tageblatt 1903, Nr. 99 u. 101.

2) Johannes KeBlers Sabbata mit kleineren Schriften und Briefen, herausgegeben vom Historischen Verein des Kantons St. Gallen, St. Gallen 1902, S. IX. Der Bericht beginnt: 3 moniales ex claustro Friburgensi Misnae profugae Uuittenbergam veniunt, ad quos [!] Lutherus epistulam parenetican (misit). Harum unam duxit in uxorem Franciscus Lanpertus. Die aus dem Freiberger Kloster entflohenen drei Nonnen, Herzogin Ursula von Münsterberg, Dorothea Tanbergin aus Freiberg und Margarethe Volckmarin aus Leipzig trafen jedoch erst am 16. Oktober 1528 in Wittenberg ein (Enders, Luthers Briefwechsel VII, 2, dazu Seidemann, Sächs. Kirchen- und Schulblatt 1879, Sp. 370). Damals aber waren Franz Lambert (der Mitte Februar 1524 Wittenberg verließ, vgl, Zeitschrift für Kirchengeschichte XXII, 130) und Johannes Keßler (der am 9. November 1523 wieder in St. Gallen eintraf, vg!. Sabbata S. X) längst wieder von Wittenberg geschieden. Dagegen ist KeBlers Anwesenheit bei der Hochzeit am 13. Juli 1523 um so glaublicher, als Lambert in Wahrheit die Magd des gleichfalls aus St. Gallen stammenden Wittenberger Mediziners Augustinus Schürpf heiratete (Zeitschr. für Kirchengesch. a. a. O.).

8) Buchwald, Zur Wittenberger Stadt- und Universitätsgeschichte in der Reformationszeit, Leipzig 1893, S. 62. zu S. 506 .

4) Köstlin, Martin Luther, 5. Aufl,, bearbeitet von G. Kawerau, II, 683, Anm. 1 
Otto Clemen.

im Jahre 1525 von einem gewissen Kaspar Gruner eine Predigt über Ev. Marci 6, 14-29 (Herodias' Tochter tanzte vor Herodes), in der der Verfasser gegen das Tanzen als sittenverderblich Stellung nimmt. ${ }^{1}$ ) Daß er dabei aus Bibelstellen wie 2. Mos. 32, 6. 19 und 1. Kön. 18, 26 Kapital zu schlagen sucht, darf uns nicht in Verwunderung setzen. Tanzende erschienen ihm wie unverständige Kinder, die mit ihren Puppen herumhüpfen und -springen; ja wenn es nicht sündiger wäre denn der Kinder Tanzen und "tocken spielen", so ginge es wohl noch hin. Besonders die Hochzeitstänze sind dem Eiferer ein Dorn im Auge; jedermann wisse, "was fur schand, spot vnd laster, fur böß gedancken, Hurerey und Ehebruch, fur zorn, neyd vnd haß, stechen, hauen vnd würgen, offt daraus entsteht".

Noch viel energischer aber hat sich später Melchior Ambach gegen das Tanzen erklärt. Er stammte aus Meiningen, wurde 1530 Pfarrer zu Neckarsteinactr, übernahm Juni 1541 die ihm bereits im Jahre vorher angetragené Prädikantenstelle zu Frankfurt a. M. und starb wahrscheinlich 1559. 2) Sein „Urteil vom Tanzen" zitiere ich nach der 1545 in Frankfurt a. $M$. bei Hermann Gülfferich erschienenen Ausgabe, in der es durch eine „Wahrhaftige Verantwortung und Widerlegung des unbescheiden, schmählichen Schreibens vom Tanzen Jacobi Ratz, Prädikanten zu Newenstadt am Koch" [d. i. Neuenstadt am Kocher im Neckarkreis] vermehrt ist. Ambach spricht sehr pessimistisch von der Sittlichkeit seiner Zeit. Saufen, Huren, Spielen und "finantzen" [d. i. wuchern, übervorteilen], ${ }^{3}$ ) mit listigen Praktiken Land und Leute beschaißen und um ihr Hab und Gut bringen wird von vielen in dieser Welt entweder als keine oder doch nur geringe Sünde geachtet, ja als besondere Kunst geübt und gelobt. Wer diese Stücklein mit einem besonderen Geschick üben kann, wird in Ehren gehalten. Wer sich aber dieser kunstreichen Stücklein mäBigt oder entschlägt, ist ein grober, ungeschickter Tölpel, ein stoischer Mönch, der der Welt ihre Kurzweil nehmen will.

1) Ausgabe von 1525: Panzer, Annalen $2828=$ Weller, Repertorium typographicum 3423. Nachdruck von Gabriel Kantz in Altenburg 1526 (Zwickauer Ratsschulbibliothek XVI, XI, $15_{39}=X X$, VIII, 20 42 . Vgl, auch schon Unschuldige Nachrichten 1715, 587 .

2) Allgemeine deutsche Biographie I, 389f. Vgl. reuestens noch $G$. Bossert, Zeitschrift für Geschichte des Oberrheins XIX, $595 \mathrm{f}$.

3) Grim D. W. 3, 1640. 
Ebenso sind Unkeuschheit, Vollsaufen, Spielen und Tanzen an der Tagesordnung. Ambach macht sich nun anheischig, das. Tanzen in einer jedem einleuchtenden Weise als Sünde und Unrecht aus der hl. Schrift zu erweisen. Nach Röm. 14, 23 ist alles, was nicht aus dem Glauben geschieht, Sünde. Nun gründet sich aber das Tanzen auf kein Gotteswort und ist danach Sünde. Auf das Beispiel der Mirjam 2. Mos. 15, 20 und Davids 2. Sam. 6, 14 darf man nicht hinweisen, denn die tanzten, um ihre jubelnde Dankbarkeit für Gottes Wohltaten zum Ausdruck zu bringen. Auch hat David keine schöne Venus an der Hand geführt, so hat auch kein Adonis oder Kupido Mirjam vorgetanzt. Jetzt ist keine Zeit zu tanzen, sondern eher zu klagen und zu weinen. Wie kann ein Christ tanzen, so ihn täglich die Welt. von Christo abschreckt, der Teufel wie ein brüllender Löwe mit. aller Macht und Bosheit ohne Unterlaß gegen ihn anstürmt?! Und zum andern ist $z \mathrm{u}$ beherzigen die Mahnung 1. Joh. 2, $15 \mathrm{ff}$. Nun ist aber nirgends mehr Fleisches- und Augenlust als beim Tanzen. Da übt man leichtfertige hurerische Gebärden nach süBem Saitenspiel und unkeuschen Liedern, da begreift man Frauen und Jungfrauen mit unkeuschen Händen, da küßt man einander mit hurerischem Umfassen. Wie oft hat ein frommes. Weib ihre lang behaltene Ehre beim Tanzen verloren!

Mit solchen Ausschreitungen scheinen die Adeligen vorangegangen zu sein. Wenigstens eifert die Schrift "Vom geilen und gotteslästerlichen Tanzen" ${ }^{1}$ ) besonders gegen die adeligen, tollen, bübischen, unzüchtigen Tänze, die rechte Hochzeiten und Schulen des Teufels in Stadt und Land, zum höchsten verschrien. und wie die Saufgelage zum bösesten Exempel für das gemeine. Volk wären. Ähnlich erschreckende Einblicke in die beim Tanzen eingerissene Wildheit und Sittenlosigkeit gewähren die von einem anderen Prediger im Jahre 1567 gegebenen Mitteilungen. Da. werden Tänze beschrieben, bei denen den Dirnen und Mägden die. Röcke bis über den Gürtel, ja über die Köpfe flögen; auch Tänzeim bloßen Hemde werden erwähnt. Der Verfasser sagt, er habe sich oft krank dagegen gepredigt, es sei jedoch alles vergeblich. ${ }^{2}$ )

1) Zitiert bei Joh. Janssen, Geschichte des deutschen Volkes seit dem Ausgange. des Mittelalters VIII, 1. bis 12. Aufl., Freiburg i. Br. 1894, S. 228.

2) Ebenda S. 445 . 